\title{
Data-driven Parameterized Model Order Reduction Using z-domain Multivariate Orthonormal Vector Fitting Technique
}

Francesco Ferranti, Dirk Deschrijver, Luc Knockaert and Tom Dhaene

\begin{abstract}
Efficient real-time design space exploration, design optimization and sensitivity analysis call for Parameterized Model Order Reduction (PMOR) techniques to take into account several design parameters, such as geometrical layout or substrate characteristics, in addition to time or frequency. This chapter presents a robust multivariate extension of the z-domain Orthonormal Vector Fitting technique. The new method provides accurate and compact rational parametric macromodels based on numerical electromagnetic simulations or measurements in either frequencydomain or time-domain. The technique can be seen as a data-driven PMOR method.
\end{abstract}

\section{Introduction}

Nowadays, full-wave electromagnetic methods [9],[17],[11] are widely used to simulate a variety of complex electromagnetic systems and are considered to be essential for efficient design. The use of these methods usually results in the computation of a huge number of field $(\mathrm{E}, \mathrm{H})$ or circuit $(\mathrm{i}, \mathrm{v})$ unknowns, in the frequency-domain or time-domain, although users are usually only interested in a few of them at the input and output ports. These methods provide high accuracy, often at a significant cost in terms of memory storage and computing time. Therefore, Model Order Reduction (MOR) techniques are crucial to reduce the complexity of the model defined by the full-wave numerical method and the computational cost required by simulations, while retaining the important physical features of the original system [7],[3].

F. Ferranti, L. Knockaert, and T. Dhaene are with the Department of Information Technology (INTEC), Ghent University-IBBT, Ghent 9000, Belgium (e-mail: francesco.ferranti@intec.ugent.be; luc.knockaert@intec.ugent.be; tom.dhaene@intec.ugent.be).

D. Deschrijver is with the Department of Information Technology (INTEC), Ghent UniversityIBBT, Ghent 9000, Belgium. He is also a post-doctoral research fellow of FWO Vlaanderen (email: dirk.deschrijver@intec.ugent.be). 
Efficient real-time design space exploration, design optimization and sensitivity analysis require the development of accurate parametric broadband macromodels that approximate the dynamic behavior of a system characterized by several design parameters, such as geometrical layout or substrate characteristics, in addition to time or frequency. These applications call for Parameterized Model Order Reduction (PMOR) techniques.

A frequency-domain technique called Multivariate Orthonormal Vector Fitting (MOVF) was presented in [4], to compute accurate rational parametric macromodels, based on parameterized frequency responses with a highly dynamic behavior. This technique can be seen as a data-driven PMOR method. Instead of reducing the size of the matrices of a parameterized state-space model directly (model-based PMOR), MOVF builds rational parametric macromodels with a reduced model complexity based on a set of input-output data samples. The goal of the macromodeling algorithm is to find a multivariate rational function which approximates a large set of $K+1$ data samples $\left\{(s, \mathbf{g})_{k}, H(s, \mathbf{g})_{k}\right\}_{k=0}^{K}$ in a least-squares sense. These data samples depend on the complex frequency $s=j \omega$ and several additional parameters $\mathbf{g}=\left(g^{(n)}\right)_{n=1}^{N}$ as design variables which describe e.g. the metallizations in an EM circuit (lengths, widths,...) or the substrate features (thickness, dielectric constant, losses, ...). The proposed approach results in accurate and compact rational parametric macromodels of complex electromagnetic systems. A generalization of MOVF to include parameter derivatives in the modeling process was proposed in [6]. $\mathrm{Pa}$ rameter derivatives provide additional information about the underlying system and can often be simulated at a significantly lower computational cost than additional samples [13],[5],[20],[15]. The inclusion of derivatives can be useful to reduce the required amount of data samples, while preserving the accuracy of the results. In this chapter a new technique, the z-domain Multivariate Orthonormal Vector Fitting (ZD-MOVF) is described, representing the $z$-domain counterpart of [4]. It is a robust multivariate extension of the $z$-domain Orthonormal Vector Fitting technique (ZDOVF) proposed in [16],[14],[21]. A microstrip example confirms the ability of the new algorithm to build parametric macromodels of dynamic systems with a good accuracy.

\section{Background}

In this section we explain the generation of z-domain data starting from frequencydomain or time-domain data and the choice of the $\lambda$ parameter of the Tustin transform. 


\subsection{Generation of z-domain data}

Microwave circuits and components can be characterized in frequency-domain or time-domain by numerical electromagnetic simulations or measurements. To obtain the corresponding parameterized $\mathrm{z}$-domain response, $H_{d}(z, g)$, where $z$ is the complex discrete frequency variable and $g$ is a real design variable, a Tustin (bilinear) transform:

$$
s \longrightarrow z=\frac{\lambda+s}{\lambda-s}, \quad \lambda \in \mathbb{R}^{+}
$$

can be used starting from frequency-domain data $H_{c}(s, g)$ :

$$
H_{c}(s, g) \longrightarrow H_{d}\left(z=\frac{\lambda+s}{\lambda-s}, g\right)
$$

where $c$ stands for continuous and $d$ for discrete. If time-domain data is available, under the hypothesis of a negligible or absent aliasing in the sampling process, the frequency response of a continuous-time system can be computed by applying standard techniques, such as Fast Fourier Transform (FFT) algorithms on the data samples:

$$
h_{d}([n], g)=h_{c}\left(n T_{s}, g\right)
$$

where the real sequence $h_{d}([n], g)$ is equal to the signal in the time domain $h_{c}(t, g)$ at the equally spaced time samples $n T_{s}$ and $T_{s}$ is the sampling period. Once the parameterized frequency response is computed, the Tustin transform (1) is used as before. The obtained z-domain data can be normalized by discrete frequency $z$ [14]. Once the parametric macromodel is computed in the $\mathrm{z}$-domain, it can be converted back to the s-domain by using inverse Tustin tranform.

\subsection{Choice of $\lambda$ of the Tustin transform}

The $\lambda$ parameter of the Tustin transform can be freely chosen [19] under the constraint that it is not a real pole of the continuous-time system [1]. The numerical example in this letter shows that the algorithm is robust with respect to an arbitrary choice of $\lambda$, since its value does not affect the accuracy of the results over a wide range. To avoid harmful numerical conditions, extreme values of $\lambda$ have to be discarded, such as very low (near zero) or very high (near infinity). 


\section{Parametric Macromodeling}

To simplify the notation, the algorithm is only described for bivariate systems. The extension to the full multivariate formulation is straightforward. As in [4], the ZDMOVF algorithm proposes to represent the parametric macromodel as the ratio of a bivariate numerator and denominator

$$
R(z, g)=\frac{N(z, g)}{D(z, g)}=\frac{\sum_{p=0}^{P} \sum_{v=0}^{V} c_{p v} \chi_{p}(z) \psi_{v}(g)}{\sum_{p=0}^{P} \sum_{v=0}^{V} \tilde{c}_{p v} \chi_{p}(z) \psi_{v}(g)}
$$

where $P$ and $V$ represent the maximum order of the corresponding basis functions $\chi_{p}(z)$ and $\psi_{v}(g)$ in the complex discrete frequency variable $z$ and the real design variable $g$, respectively. To establish the coefficients $c_{p v}$ and $\tilde{c}_{p v}$ of numerator and denominator in (4), the ZD-MOVF algorithm minimizes the Sanathanan-Koerner (SK) cost function [18] on a set of $K+1$ data samples $\left\{(z, g)_{k}, H_{d}(z, g)_{k}\right\}_{k=0}^{K}$. SK is an iterative procedure, in the first iteration step of the algorithm $(t=0)$ Levi's cost function [12] is minimized to obtain an initial estimate of the coefficients $c_{p v}$ and $\tilde{c}_{p v}$. In the following steps $(t=1, . ., T)$ of the SK iteration, the inverse of the previously estimated denominator $D^{(t-1)}(z, g)$ is used as an explicit least-squares weighting factor. A relaxed non-triviality constraint is added as an additional row in the system matrix [8], to avoid the trivial null solution and improve the convergence of the algorithm. Each equation is split in its real and imaginary parts, to ensure that the model coefficients $c_{p v}^{(t)}, \tilde{c}_{p v}^{(t)}$ are real. Scaling each column to unity length [7] is suitable to improve the numerical accuracy of the results.

\section{Choice of basis functions}

In this section we describe the choice of the basis functions for the discrete frequency and other parameters.

\subsection{Discrete Frequency-Dependent Basis Functions}

Based on a prescribed set of stable poles $\mathbf{a}=\left\{-a_{p}\right\}_{p=1}^{P}$, a set of partial fractions $\chi_{p}(z, \mathbf{a})$ is chosen, with $\chi_{0}(z)=1$. To select the poles two steps are followed: first, they are chosen in the s-domain as complex conjugate pairs with small real parts and the imaginary parts linearly spaced over the frequency range of interest [7] and after that, the Tustin transform (1) is applied to map them from s- to z-domain. A linear combination of two fractions is chosen to ensure that the residues of $\chi_{p}(z, \mathbf{a})$ and $\chi_{p+1}(z, \mathbf{a})$ come in perfect conjugate pairs leading to real-valued time domain responses, i.e. : 


$$
\begin{aligned}
\chi_{p}(z, \mathbf{a}) & =z\left(z+a_{p}\right)^{-1}+z\left(z+a_{p+1}\right)^{-1} \\
\chi_{p+1}(z, \mathbf{a}) & =j z\left(z+a_{p}\right)^{-1}-j z\left(z+a_{p+1}\right)^{-1}
\end{aligned}
$$

To improve the numerical stability of the modeling algorithm, the Takenaka-Malmquist basis functions [10] can be used, as shown in [16]:

$$
\begin{gathered}
\chi_{p}(z, \mathbf{a})=k_{p}\left(\prod_{i=1}^{p-1} \frac{1+a_{i}^{*} z}{z+a_{i}}\right) \frac{(1-z)\left|1-a_{p}\right|}{\left(z+a_{p}\right)\left(z+a_{p+1}\right)} \\
\chi_{p+1}(z, \mathbf{a})=k_{p+1}\left(\prod_{i=1}^{p-1} \frac{1+a_{i}^{*} z}{z+a_{i}}\right) \frac{(1+z)\left|1+a_{p}\right|}{\left(z+a_{p}\right)\left(z+a_{p+1}\right)}
\end{gathered}
$$

where

$$
k_{p}=k_{p+1}=\frac{\sqrt{2}}{2} \sqrt{1-\left|a_{p}\right|^{2}}
$$

The orthonormal basis functions can improve the conditioning of the system equations and are less sensitive to the choice of the initial poles. Their use ensures a more numerically robust macromodeling procedure [3].

\subsection{Parameter-Dependent Basis Functions}

The parameter-dependent basis functions $\psi_{v}(g, \mathbf{b})$ are also chosen in partial fraction form as a function of $j g$, hence in rational form. The set of starting poles $\mathbf{b}=\left\{-b_{v}\right\}_{v=1}^{V}$ is composed by complex pairs with small real parts of opposite sign and imaginary parts linearly spaced over the parameter range of interest, provided that $\psi_{0}(g)=1$. A linear combination of two fractions is used to ensure that $\psi_{v}(g, \mathbf{b})$ and $\psi_{v+1}(g, \mathbf{b})$ are real functions [4]:

$$
\begin{aligned}
\psi_{v}(g, \mathbf{b}) & =\left(j g+b_{v}\right)^{-1}-\left(j g-\left(b_{v}\right)^{*}\right)^{-1} \\
\psi_{v+1}(g, \mathbf{b}) & =j\left(j g+b_{v}\right)^{-1}+j\left(j g-\left(b_{v}\right)^{*}\right)^{-1}
\end{aligned}
$$

\section{Example: Double folded stub microstrip bandstop filter}

The double folded stub microstrip bandstop filter [2] under study is shown in Figure 1. The substrate is $0.1270 \mathrm{~mm}$ thick with a relative dielectric constant $\varepsilon_{r}$ equal to 9.9. The scattering parameters of the system are simulated by ADS-Momentum ${ }^{1}$ in the s-domain and subjected to (2), to obtain the corresponding parametrized z-domain response.

\footnotetext{
${ }^{1}$ Momentum EEsof EDA, Agilent Technologies, Santa Rosa, CA.
} 


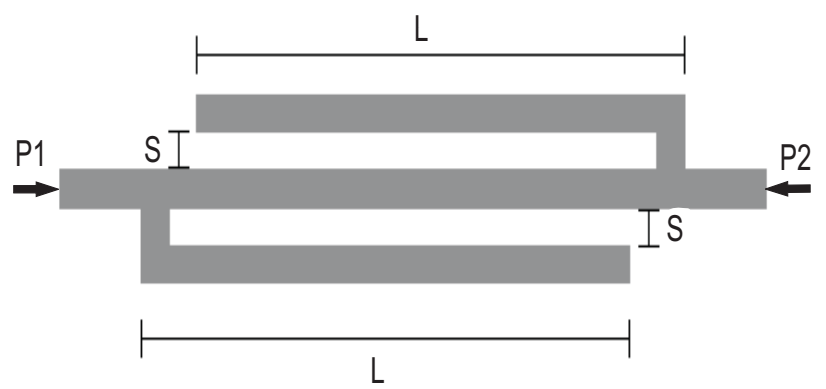

Fig. 1 Geometry of the double folded stub microstrip bandstop filter [2].

The parametric macromodels of scattering parameters $S_{11}$ and $S_{21}$ are built as functions of the varying length of each folded segment $L \in[1.98 \mathrm{~mm}-2.40 \mathrm{~mm}]$ and varying spacing between a folded stub and the main line $S \in[0.061 \mathrm{~mm}-0.243$ $\mathrm{mm}]$ over the frequency range $[5 \mathrm{GHz}-20 \mathrm{GHz}]$. The desired model accuracy is set to $-60 \mathrm{~dB}$, which corresponds to 3 significant digits. The initial data grid for $S_{11}$ and $S_{21}$ is of size $14 \times 10 \times 22$ samples $(L, S$, freq). The corresponding number of poles is chosen 6, 4 and 10 for both scattering parameters. Figure 2 and Figure 3 show the magnitude of the trivariate macromodels of $S_{11}$ and $S_{21}$ for the minimum and maximum values of the spacing variable $S$.

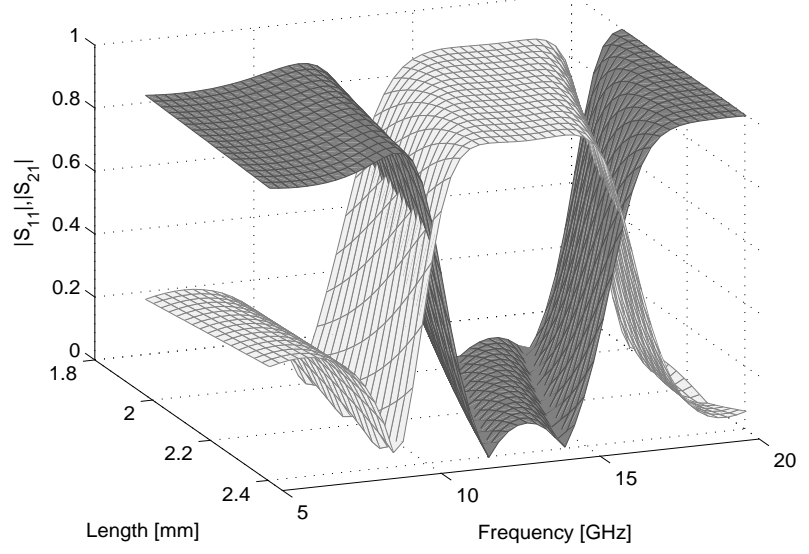

Fig. 2 Magnitude of the trivariate macromodels of $S_{11}$ (light grey surface) and $S_{21}$ (dark grey surface) for $S=0.061 \mathrm{~mm}$. 


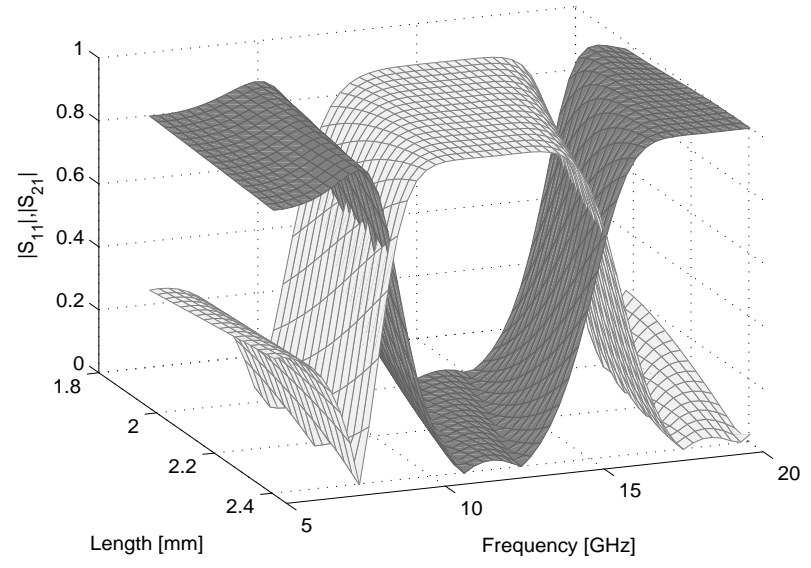

Fig. 3 Magnitude of the trivariate macromodels of $S_{11}$ (light grey surface) and $S_{21}$ (dark grey surface) for $S=0.243 \mathrm{~mm}$.

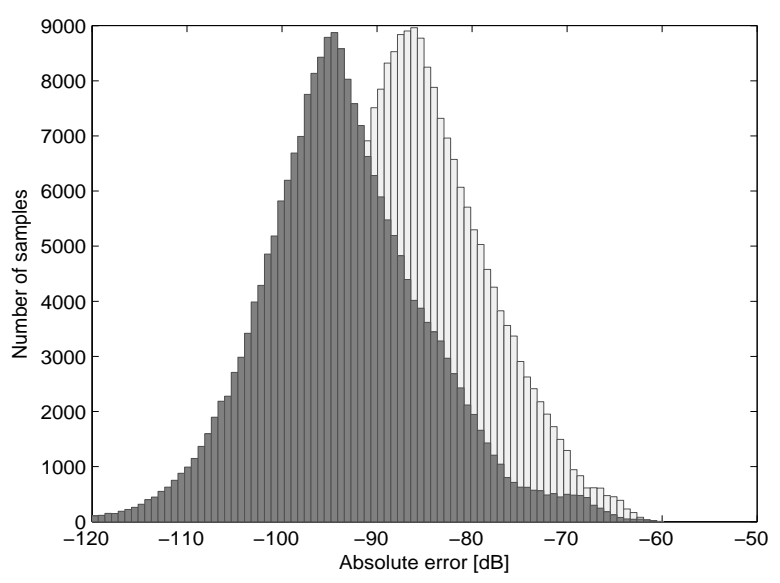

Fig. 4 Histogram : error distributions of the trivariate macromodels of $S_{11}$ (light grey) and $S_{21}$ (dark grey) over 226500 validation samples.

To compute the macromodels only 4 and 3 iterations of SK method discussed in Section 3 are needed and the maximum absolute error in the initial data grid corresponds to $-62.84 \mathrm{~dB}$ and $-67.54 \mathrm{~dB}$, respectively. To confirm the quality of built macromodels a set of validation data samples is computed on a very dense grid of size $50 \times 30 \times 151$ samples. The histogram in Figure 4 shows the number of validation samples that corresponds to a certain absolute error for both trivariate macromodels. Figure 4 shows that they have a good overall accuracy and the max- 
imum absolute error over all the validation samples is bounded by $-60.17 \mathrm{~dB}$ and $-61.06 \mathrm{~dB}$ for $S_{11}$ and $S_{21}$ respectively. The choice of the $\lambda$ parameter in the Tustin transform (1) does not influence the model accuracy over a broad range of values $\left[10^{-3}-10^{23}\right]$. It confirms that $\lambda$ is free to choose and illustrates the robustness of the algorithm.

\section{Conclusions}

This chapter presents a robust multivariate extension of the z-domain Vector Fitting technique [16],[14],[21], for the calculation of accurate and compact parametric macromodels of high-speed components. By combining rational basis functions and the Sanathanan-Koerner least-squares estimator, the robustness of the method is ensured. An example illustrates the capability of the algorithm to model dynamic parameterized frequency responses with a good accuracy. Once the multivariate macromodeling process is completed, the resulting scalable behavior model can efficiently be employed in real-time design space exploration, fast optimization and sensitivity analysis.

Acknowledgements This work was supported by a grant of the Research Foundation-Flanders (FWO-Vlaanderen).

\section{References}

1. U. M. Al-Saggaf and G. F. Franklin, "Model Reduction Via Balanced Realizations: An Extension and Frequency Weighting Techniques", IEEE Trans. on Automatic Control, vol. 33, no. 7, pp. 687-692, July 1988.

2. J. W. Bandler, R. M. Biernacki, S. H. Chen, P. A. Grobelny, and R. H. Hemmers, "Space mapping technique for electromagnetic optimization", IEEE Trans. on Microwave Theory and Techniques, vol. 42, no. 12, pp. 2536-2544, December 1994.

3. D. Deschrijver, B. Haegeman and T. Dhaene, "Orthonormal Vector Fitting: A Robust Macromodeling Tool for Rational Approximation of Frequency Domain Responses", IEEE Trans. on Advanced Packaging, vol. 30, no. 2, pp. 216-225, May 2007.

4. D. Deschrijver, T. Dhaene and D. De Zutter, "Robust Parametric Macromodeling using Multivariate Orthonormal Vector Fitting", IEEE Trans. on Microwave Theory and Techniques, vol. 56, no. 7, pp. 1661-1667, July 2008.

5. T. Dhaene and D. Deschrijver, "Generalised Vector Fitting Algorithm for Macromodelling of Passive Electronic Components ”, IEE Electronics Letters, vol. 41, no. 6, pp. 299-300, March 2005.

6. F. Ferranti, D. Deschrijver, L. Knockaert and T. Dhaene, "Fast Parametric Macromodeling of frequency responses using parameter derivatives", IEEE Microwave and Wireless Components Letters, vol. 18, no. 12, pp. 761-763, December 2008.

7. B. Gustavsen and A. Semlyen, "Rational Approximation of Frequency Domain Responses by Vector Fitting", IEEE Trans. on Power Delivery, vol. 14, no. 3, pp. 1052-1061, July 1999.

8. B. Gustavsen, "Improving the Pole Relocating Properties of Vector Fitting", IEEE Trans. on Power Delivery, vol. 21, no. 3, pp. 1587-1592, July 2006. 
9. R. F. Harrington, Field Computation by Moment Methods. NewYork: Macmillan, 1968.

10. P. S. C. Heuberger, P. M. J. Van den Hof and B. Wahlberg, Modelling and Identification with Rational Orthogonal Basis Functions, Springer-Verlag London, 2005.

11. J. M. Jin, The Finite Element Method in Electromagnetics, 2nd ed. New York: Wiley, 2002.

12. E. C. Levi, "Complex Curve Fitting", IRE Trans. on Automatic Control, vol. AC-4, pp. 37-44, May 1959.

13. P. Liu, Z-F. Li and G-B. Han, "Application of Asymptotic Waveform Evaluation to Eigenmode Expansion Method for Analysis of Simultaneous Switching Noise in Printed Circuit Boards (PCBs)", IEEE Transactions on Electromagnetic Compatibility, vol. 48, no. 3, pp. 485-492, August 2006.

14. Y. S. Mekonnen and J. E. Schutt-Ainé, "Broadband Macromodeling of Sampled Frequency Data Using z-domain Vector Fitting", IEEE Workshop on Signal Propagation on Interconnects, SPI 2007, Camogli Genova (Italy), pp. 45-48, May 2007.

15. N. K. Nikolova, Y. Li, Y. Li and M. H. Bakr, "Sensitivity Analysis of Scattering Parameters with Electromagnetic Time-Domain Simulators", IEEE Transactions on Microwave Theory and Techniques, vol. 54, no. 4, pp. 1598-1610, April 2006.

16. B. Nouri, R. Achar, M. Nakhla and D. Saraswat, " $z$-Domain Orthonormal Vector Fitting for Macromodeling High-Speed Modules Characterized by Tabulated Data", IEEE Workshop on Signal Propagation on Interconnects, SPI 2008, Avignon (France), pp. 1-4, May 2008.

17. A. E. Ruehli and P. A. Brennan, "Efficient Capacitance Calculations for Three-Dimensional Multiconductor Systems", IEEE Trans. on Microwave Theory and Techniques, vol. 21, no. 2, pp. 76-82, February 1973.

18. C. Sanathanan and J. Koerner, "Transfer Function Synthesis as a Ratio of two Complex Polynomials", IEEE Trans. on Automatic Control, vol. 8, no. 1, pp. 56-58, January 1963.

19. K. C. Sou, A. Megretski and L. Daniel, "A Quasi-Convex Optimization Approach to Parameterized Model Order Reduction", IEEE Trans. on Computer-Aided Design of Integrated Circuits and Systems, vol. 27, no. 3, pp. 456-469, March 2008.

20. J. Ureel and D. De Zutter, "A New Method for Obtaining the Shape Sensitivities of Planar Microstrip Structures by a Full-Wave Analysis ", IEEE Transactions on Microwave Theory and Techniques, vol. 44, no. 2, pp. 249-260, February 1996.

21. N. Wong and C. Lei, "IIR Approximation of FIR Filters Via Discrete-Time Vector Fitting", IEEE Trans. on Signal Processing, vol. 56, no. 3, pp. 1296-1302, March 2008. 